Original Research Paper

\title{
The Multiple Tuned Mass Damper for Passive Control of Structures by Cuckoo Algorithm
}

\author{
${ }^{1}$ Hamid Jafari Nia, ${ }^{1}$ Hamid Reza Babaali, \\ ${ }^{1}$ Amir Hossein Heidari and ${ }^{2 *}$ Kaveh Ostad-Ali-Askari \\ ${ }^{I}$ Department of Civil Engineering, Khorramabad Branch, Islamic Azad University, Khorramabad, Iran \\ ${ }^{2}$ Department of Water Engineering, College of Agriculture, Isfahan University of Technology, Isfahan 84156-83111, Iran
}

\author{
Article history \\ Received: 06-01-2020 \\ Revised: 15-07-2020 \\ Accepted: 15-07-2020 \\ Corresponding Author: \\ Kaveh Ostad-Ali-Askari \\ Department of Water \\ Engineering, College of \\ Agriculture, Isfahan University \\ of Technology, Isfahan \\ 84156-83111, Iran \\ Email: ostadaliaskari.k@of.iut.ac.ir \\ kaveh.oaa2000@gmail.com
}

\begin{abstract}
Adjustable mass dampers are recently used to reduce seismic vibrations of engineering structures. Function of these types of systems requires a suitable setting of their parameters. In this study, a practical method to design Multiple Tuned Mass Dampers (MTMD) has been proposed to minimize the maximum displacement of roof, acceleration of roof and TMDs displacement to reduce the seismic response of structures. To solve the problem Cuckoo Optimization Algorithm (CMOA) has been used. The results of numerical simulations for a shear frame of ten floors exposed to earthquakes demonstrate that CMOA is able to present appropriate solutions in the form of Parato charts for setting optimal parameters of MTMD, caused by creating a proper compromise between the objective functions in conflict with each other. Eventually, MTMD desirably reduces seismic responses of structures. Also, MTMD performance depends on input quake, TMD mass ratio and the number of TMDs.
\end{abstract}

Keywords: Passive Control of Structures, Multiple Tuned Mass Dampers, Multi-Objective Optimization, Cuckoo Algorithm, Tuned Mass Dampers, Optimal Parameters

\section{Introduction}

Tuned Mass Damper (TMD) is one of the passive means of energy dissipation. By absorbing some of the input energy from the dynamic load to the structure, this tool reduces the demand for energy dissipation in the main structure (Hoang and Warnitchai, 2005).

MTMD is made up of several single TMDs. These dampers can be divided and designed into two forms: Parallel and series and be installed in the structure; moreover, they can be used in a structural system for both integrated (integrated in one place) or distributed mode (distributed in structures space) (Ohtori et al., 2004).

Surveys depicted that MTMD function depends on the mass value, the number of TMDs, frequency range and the quality of their distribution (Kareem and Kline, 1995). MTMD can be adjusted to different modes and dampers are placed in different positions in the main structure to enhance seismic performance. According to the light weight of the dampers in the system, improper use of any TMD setting will not cause destructive effects on the structural seismic response. In addition, MTMD performance is less sensitive to the uncertainty of system parameters (Yamaguchi and Harnpornchai, 1993), (Abé and Fujino, 1994).

The application of a TMD on tall buildings practically, there may be the need to a heavy mass and substantial space to install it. Designing a TMD, for very tall buildings that in higher modes may play a significant role in the overall response, may have little effect on the control response of higher modes just for the first mode of vibration. To solve the problem, the use of MTMD instead of a single TMD is suggested. To design MTMD, several methods have been used. In the early stages of designing MTMD to simplify the analysis and design process, some design constraints such as mass and damping ratios have been considered as known for TMD. Igusa and Xu (1994), aiming to generalize optimal design issue, ignored the TMD's mass and damping ratios in design and have proposed an asymptotic analysis for designing MTMD for a SDOF structure influenced by wind power. Jangid (1999) suggested a method for determining the optimal MTMD parameters for a not damped system under harmonic excitation bid. This method was based on minimizing the stable movement of the original system by using numerical study techniques. 
In a study proposed by (Wu and Chen, 2000) MTMD had been divided into several groups, that each one was related to a mode and included multiple dampers distributed in different floors. As a result, the acceleration of the structures reduced to optimal minimum by MTMD. Li (2002) evaluated the performance of five MTMD makeups that included different combinations of parameters of the TMD (mass, stiffness and damping). To study the effectiveness and capability of MTMD (Dehghan-Niri et al., 2010) used a general optimization method for designing MTMD systems to reduce response of unstable structure under harmonic excitation. Moon (2010) examined distributed MTMD efficiency vertically along with the height of a building. (Tigli, 2012) studied linear dampers system subject to random loads and presented a formula. It has proposed an efficient method for designing MTMD to reduce the seismic response of structures based on the definition of an optimization problem (GA) where TMDs' parameters are considered as variables and minimizing the greatest structural response is considered as a function.

In this study, the optimization problem is defined with the aim of setting optimal parameters of MTMD and to solve it CMOA is used. MTMD parameters are considered as design variables. Since set the parameters of MTMD is basically a multi-objective optimization problem, it is defined in single, two, three-objective modes and MTMD optimal parameters for each mode is determined. In this study, besides designing multiple mass damper using the proposed method, the impact of various factors such as the number of TMDs, mass percentage, record of different earthquakes is investigated in MTMD.

\section{Equation of Structure Motion Equipped with MTMD}

Dynamic equation for an $n$ degree of freedom shear structure with linear behavior, under $\ddot{U}_{g}(t)$ basic acceleration and $N_{T M D}$ number of single mass dampers with different dynamic characteristics, where the dampers are installed on the last floor of the structure and in parallel is expressed as follows:

$M \ddot{x}(t) C \dot{x}(t)+K x(t)=-[M] e \ddot{u}_{g}(t)$

where, mass, damping and stiffness matrixes are obtained as follows.

$$
\begin{aligned}
& M=\left[\begin{array}{cccccccccc}
m_{1} & 0 & \ldots & 0 & 0 & 0 & 0 & \ldots & 0 & 0 \\
0 & m_{2} & \ldots & 0 & 0 & 0 & 0 & \ldots & 0 & 0 \\
\vdots & \vdots & \ddots & \vdots & \vdots & \vdots & \vdots & \vdots & \vdots & \vdots \\
0 & 0 & \ldots & m_{n-1} & 0 & 0 & 0 & \ldots & \vdots & \vdots \\
0 & 0 & \ldots & 0 & m_{n} & 0 & 0 & \ldots & 0 & 0 \\
0 & 0 & \ldots & 0 & 0 & m_{d_{1}} & 0 & \ldots & 0 & 0 \\
0 & 0 & \ldots & 0 & 0 & 0 & m_{d_{2}} & \ldots & 0 & 0 \\
\vdots & \vdots & \ldots & \vdots & \vdots & \vdots & \vdots & \ddots & \vdots & \vdots \\
0 & 0 & \ldots & 0 & 0 & 0 & 0 & \ldots & m_{d_{N_{T M D}-1}} & 0 \\
0 & 0 & \ldots & 0 & 0 & 0 & 0 & \ldots & 0 & m_{d_{N_{T M D}}}
\end{array}\right]
\end{aligned}
$$

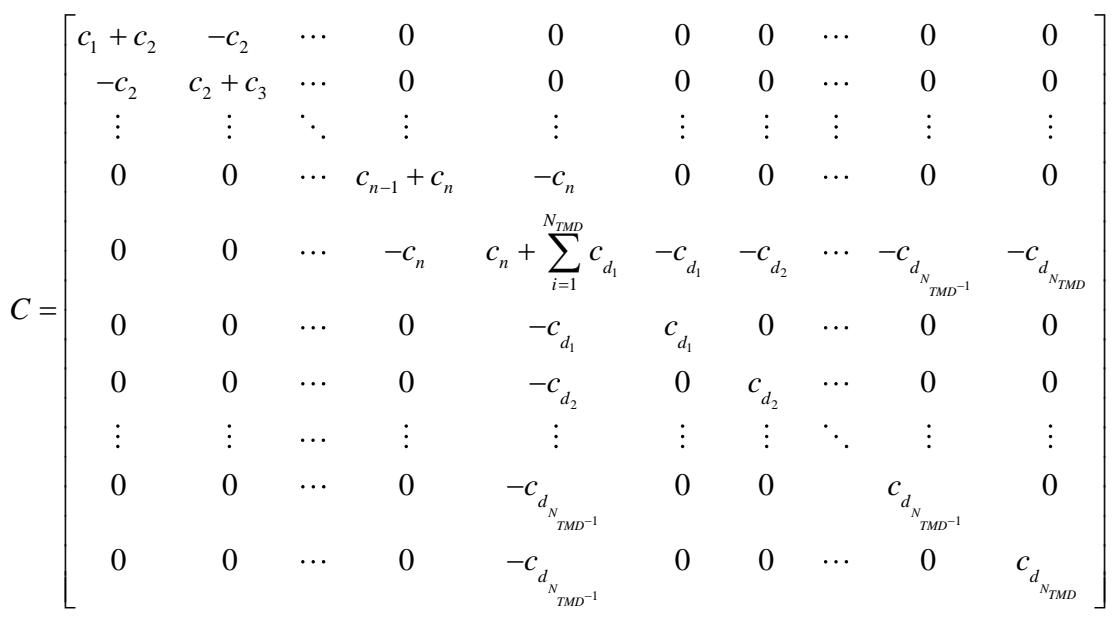




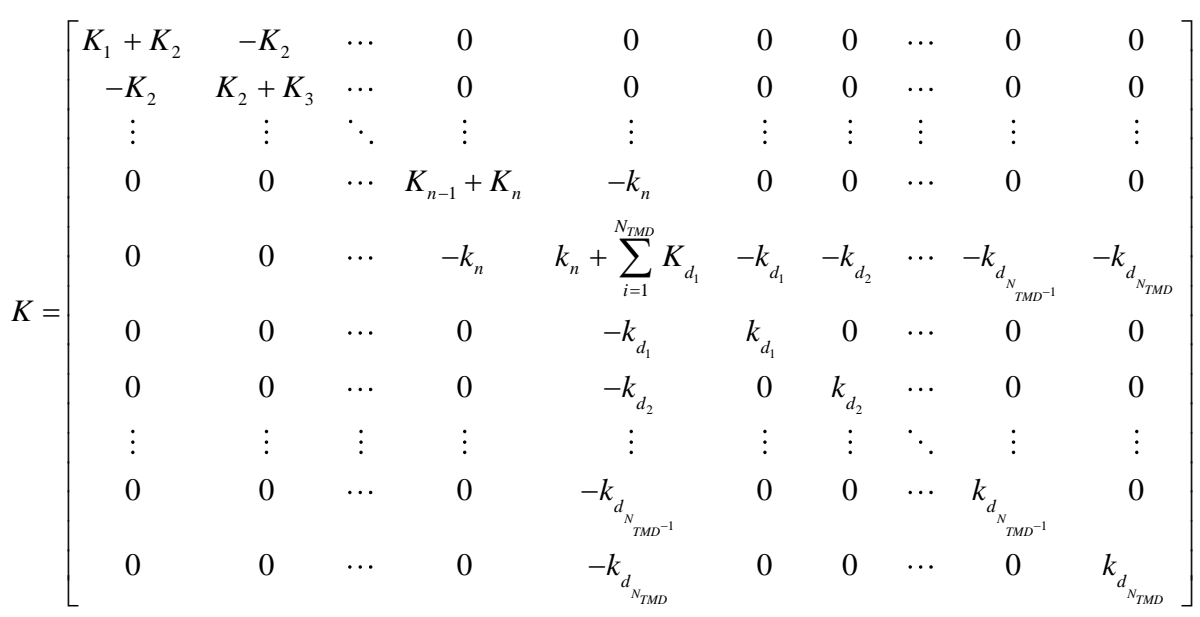

where, in the above equation, $e^{t}=[-1-1-1 \ldots-1]_{1 \times\left(n+N_{T M D}\right)}$ the momentum transfer vector $\ddot{U}_{g}(t)$ to the masses $x(t), \dot{x}(t)$ and $\ddot{x}(t)$ expressing displacement, velocity and acceleration of the floor vectors in adjustable mass dampers (Tigli, 2012).

\section{Introducing Cuckoo Optimization Algorithm}

Cuckoo search is a stochastic metaheuristic algorithm that models the obligate brood parasitism of some cuckoo birds. CS has been introduced by (Yang and Deb, 2009) to solve optimization problems. Two important features in this algorithm make CS superior to many other metaheuristic algorithms. First, having infinite mean and variance, Lévy flights can explore the search space better than standard Gaussian processes. Secondly, CS restores a balance between local search and global search exploration. Some studies showed that CS provides a better performance than GA and PSO in terms of simplicity of algorithms, having less parameter to tune and higher speed (Rajabioun, 2011), (Gandomi et al., 2013). Also, a comparison between $\mathrm{CS}, \mathrm{PSO}$ and $\mathrm{ACO}$ shows that $\mathrm{CS}$ gives more robust results (Civicioglu and Besdok, 2013).

The population size $n$, switching probability $p a$, step-size $\alpha$ and the Lévy flights exponent $\beta$ are configurable parameters of CS. Apart from the population size, the switching probability is the key parameter of the CS. Other parameters such as the Lévy flights exponent and step-size can be set as $\alpha=$ 0.1 and $\beta=1.5$ for most problems, $p a$ and $n$ are variable and have great effects on the algorithm performance. A balance between local and global optimization is created by the switching probability. The probability for global optimization is reduced with increasing pa and vice versa (Rajabioun, 2011), (Yang and Deb, 2009).

The steps of the standard CS algorithm can be described as follows (Yang and Deb, 2009; 2014):
I. Each cuckoo lays one egg at a time and dumps it in a randomly chosen nest (crossover operator)

II. Number of nests that contain eggs with high quality will be transferred to the next generation (elitism)

III. The number of available host nests is fixed and a host can discover an alien egg with a Probability pa (mutation operator)

The first rule produces a new solution using a Lévy Flights and can be considered as a crossover operator. Outline of generating new solution via Lévy flight is summarized as below:

$X_{i}^{t+1}=X_{i}^{t}+\alpha \otimes L e^{\prime} v y(\beta)$

where, $\alpha=\alpha_{0} \otimes\left(X_{j}^{t}-X_{i}^{t}\right)$ and $L e^{\prime} v y(\beta)=u /|v| \frac{1}{\beta}$ Here, $a$ is the step size parameter, $a_{0}$ is the step size scaling factor, $X_{j}^{t}$ and $X_{i}^{t}$ are two randomly selected solutions, Lévy $(\beta)$ is the step length which is produced according the Lévy Flights and $\beta$ is Lévy flights exponent. In addition, the parameters $u$ and $v$ are given by the normal distributions as shown in Equation (5). In this equation, $G$ is the standard Gamma function.

$\sigma_{u}=\left\{\frac{\Gamma(1+\beta) \sin (\pi \beta / 2)}{\Gamma\left[\frac{1+\beta}{2}\right] \beta 2^{(\beta-1) / 2}}\right\}^{1 / \beta}$

The second rule applies elitism strategy to accelerate convergence rate of CS. Finally, the third rule incorporates probabilistic strategy to replace not so good solutions. Indeed, it can be treated as a mutation search operator and prevents algorithm of being trapped in a 
local minimum. Equation (4) indicates how a new solution can be generated by this search operator:

$$
X_{i}^{t+1}=x_{i}^{t}+r \otimes H\left(\rho_{a}-\varepsilon\right) \otimes\left(X_{j}^{t}-X_{k}^{t}\right)
$$

In which $H(u)$ represents the Heaviside function, $\epsilon$ and $r$ are some random numbers with uniform distributions and $X_{j}^{t}, X_{k}^{t}$ are two solutions that are selected randomly. The basic steps of the CS can be summarized as the pseudo code shown in Fig. 1.

\section{Numerical Example}

To evaluate the performance of the proposed method in optimum designing of MTMD and influence of TMDs parameters on structural response, a 10-story structure has been simulated with the same mass, stiffness and damping for all stories, respectively with the values of 360 tons, $650 \mathrm{MN} / \mathrm{m}$ and $6.2 \mathrm{MN}$.sec/m.

\section{Earthquake Design}

Generally, dynamic loads like earthquakes and strong winds are incidental non-predictive loads. Since the seismic events are random processes, to get acceptable results in designing, there is a need for time history analysis. However, if optimum parameters of TMDs are set for a specific earthquake, there is no guarantee that they are also effective for other earthquakes.

Carrying out these settings for several different earthquakes is time consuming and due to the large volume of outputs, making it difficult to judge how to set optimal parameters.
To fix this problem, one can use spectral density function instead of a set of time history input in random analyses. White noise is as a random process with uniform spectral-power density with $S_{0}$ velocity on the range of all frequencies. Since the turbulence created is net, to use this turbulence as the basic provocation for a structure, using a filter that makes waves on the modeling of the soil is essential. Accordingly, the artificial acceleration of land used for modeling probable earthquakes is produced with the help of Gaussian white noise with a narrow band filter known as filter models (Fan and Ahmadi, 1990).

With regard to different earthquakes near to and far from a fault with different frequency content and using least-squares fitting techniques, (Nagarajaiah and Narasimhan, 2006) proposed a modified form of KanaiTajimi filter that well models accelerated induced parameters of various earthquakes that can be used in simulation of earthquakes. Equation (5) represents the transfer function provided by them:

$F(s)=\frac{4 \xi_{g} \omega_{g} S}{S^{2}+2 \xi_{g \omega_{g}} S+\omega_{g}{ }^{2}}$

$\xi_{g}$ and $\omega_{g}$, are the damping and frequency of the ground respectively, have different soils for different values. In these studies the values $\omega_{g}=2 \pi \frac{\mathrm{rad}}{\mathrm{s}}, \xi_{g}=0,3$ that correspond to the typical soils are considered. Artificial earthquakes generated from this function, a good statistical representation of this earthquake with different intensity and frequency content.

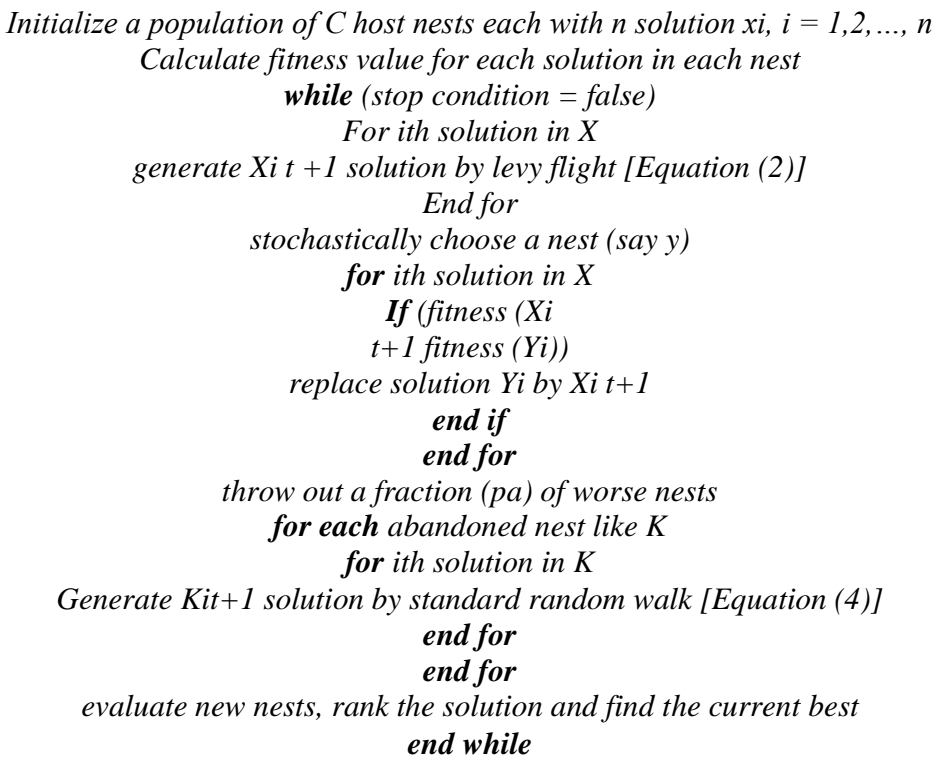

Fig. 1: Pseudo code of CS 


\section{Defining the Optimal Problem for Setting TMD Optimal Parameters}

In this study, the optimization problem with the aim of setting optimal parameters MTMD defined and to solve it cuckoo multi-objective optimization algorithm is used. MTMD setting parameters are considered as designing variables. Since setting MTMD parameters is a multiobjective optimization problem, optimization problem in single, two and three objectives are defined and MTMD optimized parameters are determined for each state. In this study, besides designing multiple mass damper using the proposed method, the impact of various factors such as the number of TMDs, the mass percentage and different earthquake records are reviewed in MTMD performance.

In reducing MTMD setting optimal parameters problem, three seismic answers including roof displacement, roof acceleration and displacement of TMD can be considered as objective functions. Given that these three objectives are in conflict with each other, therefore basically setting MTMD parameters is a multi-objective optimization problem. Multi-objective optimization methods can provide a suitable reconciliation between the objectives in conflict with each other. Considering reduction of roof displacement alone may lead to roof momentum increase, on the other hand, the reduction of TMD displacement due to physical space limitations defined for TMDs movement should be considered in the optimization. This is while, in previous studies, the problem of setting optimal parameters of TMD and MTMD is studied only with respect to one objective function. To define the optimization problem, three criteria are defined as the following:

$I_{1}-\frac{\max \left|x_{r c}\right|}{\max \left|x_{r u}\right|} I_{2}=\frac{\max \left|\ddot{x}_{r c}\right|}{\max \left|\ddot{x}_{r u}\right|} I_{3}=\frac{\max \left|x_{t m d}\right|}{\max \left|x_{r c}\right|}$

where, $I_{1}$ is maximum displacement of the roof class of the building in the structure equipped with MTMD, normalized to the maximum displacement of roof class without MTMD. The $I_{2}$ is the maximum acceleration of roof class in the structure equipped with MTMD that is normalized to the maximum acceleration of the roof class without MTMD and $I_{3}$ is the maximum displacement of MTMD normalized to the maximum displacement of the roof class equipped with MTMD.

TMD parameters get specified range of values that is defined based on the view of the designer and some practical constraints. In this research, studies of setting the optimal parameters of MTMD for a given mass ratio of TMD is done so that if $\mu$ and $M_{t o t}$ are mass percentage of TMDs and the total mass of the structure respectively, in the case of non-uniform distribution of MTMD masses, equation can be considered:

$\sum_{i=1}^{M \text { Mtmd }} m_{d_{i}}=\mu_{0} M_{\text {tot }}$
If the same mass is chosen for all selected TMDs, then:

$$
m_{d_{1}}=m_{d_{1}}=\cdots=m_{d_{N_{m a d}}}=\mu_{0} \frac{M_{t o t}}{N_{t m d}}
$$

In this case, for each predetermined $\mu$, there are four optimization problem have been defined as follows:

$$
\begin{aligned}
& \text { Find }: S=\left[c_{d_{1}}, \ldots, c_{d_{N_{\operatorname{mad}}}}, k_{d_{1}}, \ldots, k_{d_{N_{m m d}}}\right] \\
& 0 \leq C_{d_{i}} \leq C_{d_{\max }} i=1,2, \ldots, N_{t m d} \\
& 0 \leq k_{d_{i}} \leq k_{d_{\max }} i=1,2, \ldots, N_{t m d}
\end{aligned}
$$

Case1: Minimize: $I_{1}$

Case2: Minimize: $I_{1}, I_{2}$

Case3: Minimize: $I_{1}, I_{3}$

Case4: Minimize: $I_{1}, I_{2}, I_{3}$

By using multi-objective optimization algorithm for $U=0,02$ and $N_{T M D} \quad$ and $\quad K_{d \max }=4 \frac{M N}{M} \quad$ and $C_{d \max }=20 \frac{K N . S}{M}$ are done. Convergence of cuckoo algorithm in minimizing $I_{1}$ index is shown in Fig. 2. The Parato charts obtained from multi-objective optimization process in three modes in defining the optimization problem for states 2,3 and 4 , are respectively shown in Fig. 3 to 5. It is found that the Parato charts obtained from the process of multiobjective optimization produce a set of optimized solutions that based on engineering comments and practical situation of the design, the designer can choose one of the solutions to the questions as the final solution. These figures well show the scramble among defined objective function. A suitable compromise among these goals can be provided with a proper answer. Accordingly, the optimal values of the parameters set by tuned mass dampers for different defined modes of optimization problem are given in relations (10) to (13):

$$
\begin{aligned}
& S 1=\left[\begin{array}{l}
1.88,17.14,1.59,13.00,13.83,17.75,2.21, \\
8.82,1,13.53,262.29,1446.63,303.32,585.17, \\
560.1,197.22,521.1,287.6,255.73,100
\end{array}\right] \\
& S 2=\left[\begin{array}{l}
1.90,15.36,17.41,2.36,4.65,8.52,6.39,16.01, \\
1.15,1.12,268.05,283.31,1931.96,173.74, \\
422.64,1319.75,100,799.22,256.47,300.53
\end{array}\right] \\
& S 3=\left[\begin{array}{l}
1.68,3.66,3.57,7.77,3.78,19.51,1.37,18.28,18.71, \\
17.83,1344.04,421.03,283.99,284.09,260.23, \\
1761.78,461.82,672.12,210.71,235.75
\end{array}\right]
\end{aligned}
$$




$$
S 4=\left[\begin{array}{l}
7.17,2.84,13.23,19.92,19.85,18.22,13.71,7.59, \\
5.15,1.02,157.12,305.1,386.35,219.1,462.62, \\
1646.073,14.28,252.85,186.94,987.71
\end{array}\right]
$$

To evaluate the seismic behavior of structures equipped with MTMD, time history analysis of structures under study were exposed to two earthquake far from faults in El Centro 1940 and Hachino 1968 as well as two earthquake near faults in Notridge 1994 and Kobe 1995, respectively, with maximum acceleration of $0.8267 \mathrm{~g}, 0.2250 \mathrm{~g}, 0.3417$ $\mathrm{g}$ and $0.8178 \mathrm{~g}$ and the amount of seismic parameters, including maximum displacement of roof structures, roofing maximum acceleration, maximum drift of floors, maximum of base shear, RMS of roof displacement,
RMS acceleration of roof, RMS drift of floors, RMS of base shear, maximum TMD displacement and displacement of RMS and TMD displacement.

RMS of each solution represents the root mean square of the solution to evaluate the extent of structural response reduction during periodic in the risk of earthquakes. To avoid prolonged paper, the results are only given for Kobe and $\mathrm{El} \mathrm{Centro} \mathrm{earthquakes} \mathrm{and}$ given in Tables 1 and 2. Two seismic responses of TMDs including maximum displacement of TMDs and RMS of TMD displacement are listed in the tables. The corresponding percentage reduction for four different modes compared to uncontrolled (without MTMD) for the mentioned earthquakes are shown in Tables 3 and 4:

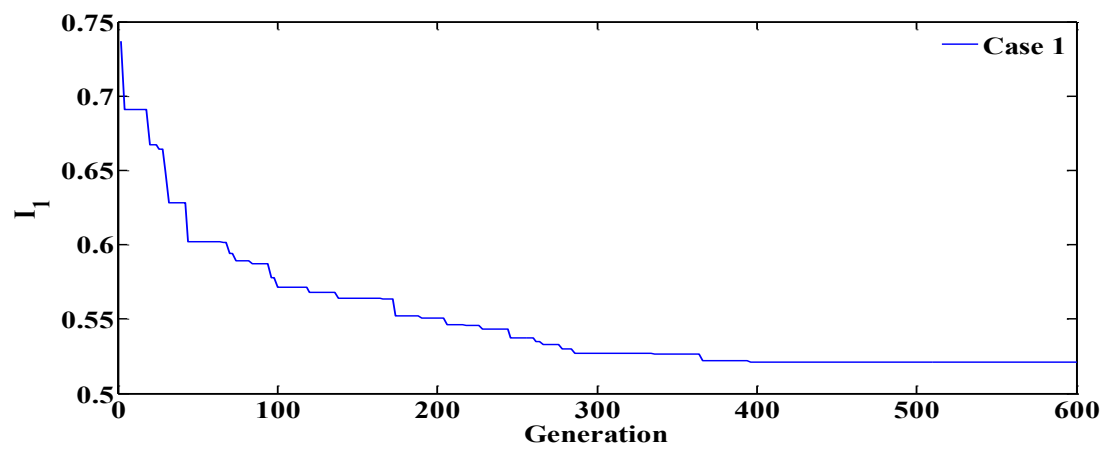

Fig. 2: Convergence Cuckoo optimization algorithms in reducing I1

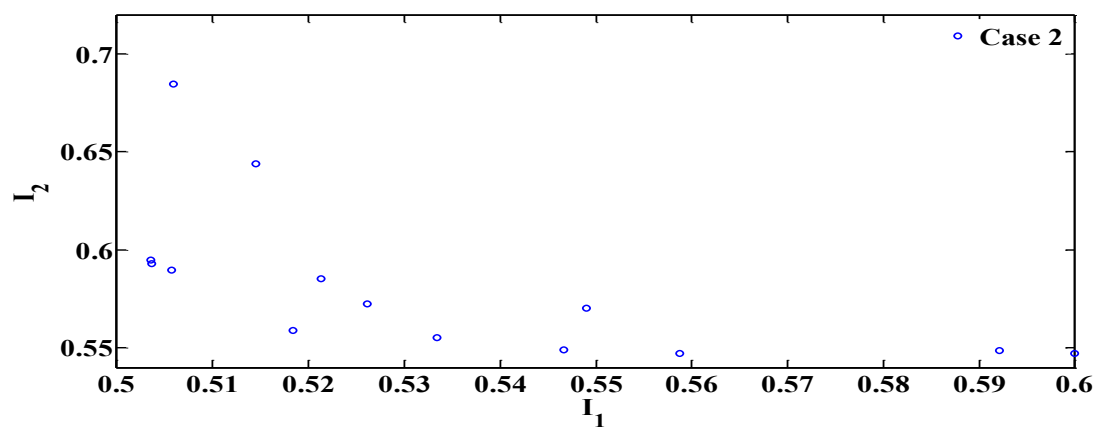

Fig. 3: Light curve in mode 2

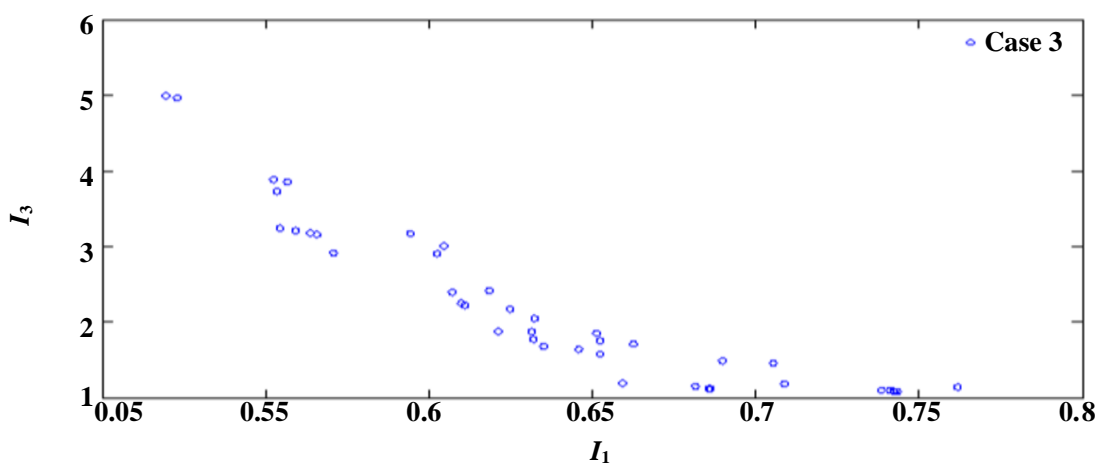

Fig. 4: Light curve in mode 3 


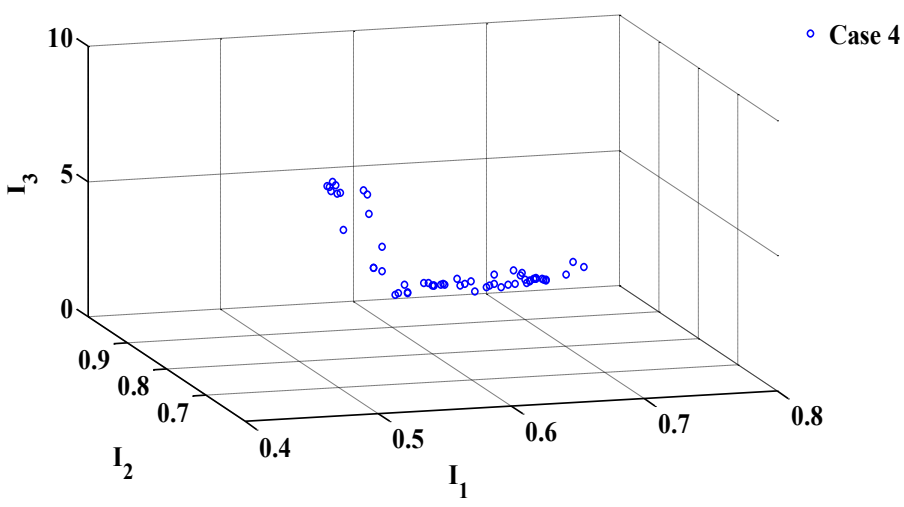

Fig. 5: Light curve in mode 4

Table 1: Seismic response of structures to earthquake in El Centro

\begin{tabular}{|c|c|c|c|c|c|}
\hline Seismic responses & Uncontrolled & Case 1 & Case 2 & Case 3 & Case 4 \\
\hline Maximum Roof Displacement (cm) & 18.78 & 12.35 & 12.60 & 12.13 & 12.02 \\
\hline Maximum Acceleration Roof $\left(\mathrm{m} / \mathrm{s}^{2}\right)$ & 8.32 & 5.94 & 5.14 & 5.94 & 5.42 \\
\hline Maximum Drift Stories $(\mathrm{cm})$ & 3.06 & 2.30 & 2.38 & 2.37 & 2.43 \\
\hline Maximum Base Shear (MN) & 19.86 & 14.72 & 15.39 & 15.15 & 15.65 \\
\hline RMS (cm) Roof Displacement & 3.53 & 2.76 & 2.87 & 2.79 & 2.69 \\
\hline Roof Acceleration RMS (m/s $\left.\mathrm{s}^{2}\right)$ & 1.48 & 1.14 & 0.98 & 1.14 & 1.13 \\
\hline Drift Stories RMS (cm) & 0.52 & 0.40 & 0.42 & 0.41 & 0.40 \\
\hline Base Shear RMS (MN) & 0.53 & 0.41 & 0.43 & 0.41 & 0.41 \\
\hline Maximum Displacement TMD (cm) & --- & 107.82 & 111.35 & 82.62 & 86.15 \\
\hline DisplacementTMD RMS (cm) & --- & 2.93 & 1.39 & 1.11 & 0.68 \\
\hline
\end{tabular}

Table 2: Seismic response of structures to earthquake in Kobe

\begin{tabular}{|c|c|c|c|c|c|}
\hline Seismic responses & Uncontrolled & Case 1 & Case 2 & Case 3 & Case 4 \\
\hline Maximum Roof Displacement(cm) & 52.68 & 47.74 & 45.75 & 46.03 & 46.00 \\
\hline Maximum Acceleration Roof $\left(\mathrm{m} / \mathrm{s}^{2}\right)$ & 23.83 & 21.78 & 21.18 & 19.16 & 21.75 \\
\hline Maximum Drift Stories $(\mathrm{cm})$ & 7.33 & 6.61 & 6.70 & 6.80 & 6.69 \\
\hline Maximum Base Shear (MN) & 47.70 & 43.18 & 42.90 & 40.68 & 42.38 \\
\hline RMS (cm) Roof Displacement & 6.03 & 3.61 & 3.76 & 3.37 & 3.88 \\
\hline Roof Acceleration RMS (m/s²) & 2.52 & 1.61 & 1.51 & 1.68 & 1.64 \\
\hline Drift Stories RMS (cm) & 0.90 & 0.54 & 0.55 & 0.57 & 0.56 \\
\hline Base Shear RMS (MN) & 0.91 & 0.58 & 0.58 & 0.61 & 0.59 \\
\hline Maximum Displacement TMD $(\mathrm{cm})$ & --- & 240.77 & 265.01 & 198.87 & 201.20 \\
\hline DisplacementTMD RMS (cm) & --- & 0.08 & 0.07 & 0.10 & 0.07 \\
\hline
\end{tabular}

Table 3: Percentage of reduction of seismic response of structures to earthquake in El Centro compared to uncontrolled state

\begin{tabular}{lcccc}
\hline \% Reduction in Seismic responses & Case 1 & Case 2 & Case 3 & Case 4 \\
\hline Maximum Roof Displacement\% Reduction in & 32.28 & 23.63 & 22.29 & 22.64 \\
\% Reduction in Maximum Acceleration Roof & 27.83 & 28.59 & 38.24 & 28.55 \\
\% Reduction in Maximum Drift Stories & 32.26 & 24.82 & 22.04 & 22.40 \\
\% Reduction in Maximum Base Shear & 32.07 & 25.87 & 22.53 & 23.71 \\
RMS \% Reduction in Roof Displacement & 23.81 & 21.76 & 18.77 & 20.99 \\
\% Reduction in Roof Acceleration RMS & 22.18 & 23.43 & 34.13 & 22.96 \\
\% Reduction in Drift Stories & 23.82 & 23.34 & 19.68 & 22.49 \\
\% Reduction in Base Shear RMS & 22.18 & 23.43 & 19.42 & 22.96 \\
\hline
\end{tabular}

Table 4: Percentage of reduction of seismic response of structures to earthquake in Kobe earthquake compared to uncontrolled

\begin{tabular}{lccrr}
\hline \% Reduction in Seismic responses & Case 1 & Case 2 & Case 3 & Case 4 \\
\hline Maximum Roof Displacement\% Reduction in & 9.37 & 13.14 & 12.61 & 12.67 \\
\% Reduction in Maximum Acceleration Roof & 8.61 & 11.14 & 19.59 & 8.73 \\
\% Reduction in Maximum Drift Stories & 9.75 & 8.53 & 7.23 & 8.63 \\
\% Reduction in Maximum Base Shear & 9.49 & 10.08 & 14.72 & 11.16 \\
RMS \% Reduction in Roof Displacement & 40.04 & 37.71 & 44.08 & 35.67 \\
\% Reduction in Roof Acceleration RMS & 35.88 & 39.86 & 33.21 & 35.05 \\
\% Reduction in Drift Stories & 40.11 & 38.78 & 36.32 & 37.26 \\
\% Reduction in Base Shear RMS & 35.88 & 35.89 & 33.21 & 35.05 \\
\hline
\end{tabular}


The results show that MTMD can appropriately reduce the seismic response of the structure. The extent of reduction is largely dependent on the input earthquake to structure, for example, MTMD have provided the most reductions in El Centro earthquake. The results show that the designer can, considering designing goals, define his optimization problem and achieve the preset optimal performance indices. For example, if optimization problem is considered to be singleobjective, MTMD are not so able to reduce the structural acceleration as they are in reducing shift of classes. However, with regard to the second mode of the problem, where deceleration of structural optimization is considered as objective function, the structure is able to properly reduce the acceleration. In addition, to reduce TMD displacement, the reduction of this response can also be considered in optimization problem to reduce the displacement of TMDs. This problem, from the standpoint of implementation issues of TMD project, is important to their move due to limitations in physical space. Figures 6 and 7 show the effect of number of TMDs and mass ratio of TMDs in reducing displacement and acceleration of structure roof by stimulation of artificial earthquake. As is seen, with increase in mass ratio of TMDs to $6 \%$, the values of $I_{1}$ and $I_{2}$ reduce and for values more than $6 \%$, the decline process has not changed a lot. It is also seen that for a fixed mass ratio increasing TMDs from 1 to 10 leads to a suitable reduction in $I_{1}$ and $I_{2}$ values, but with more increase of TMDs the response has not changed much. The amount of this being affected in structural shift is more than in deceleration of the structure.

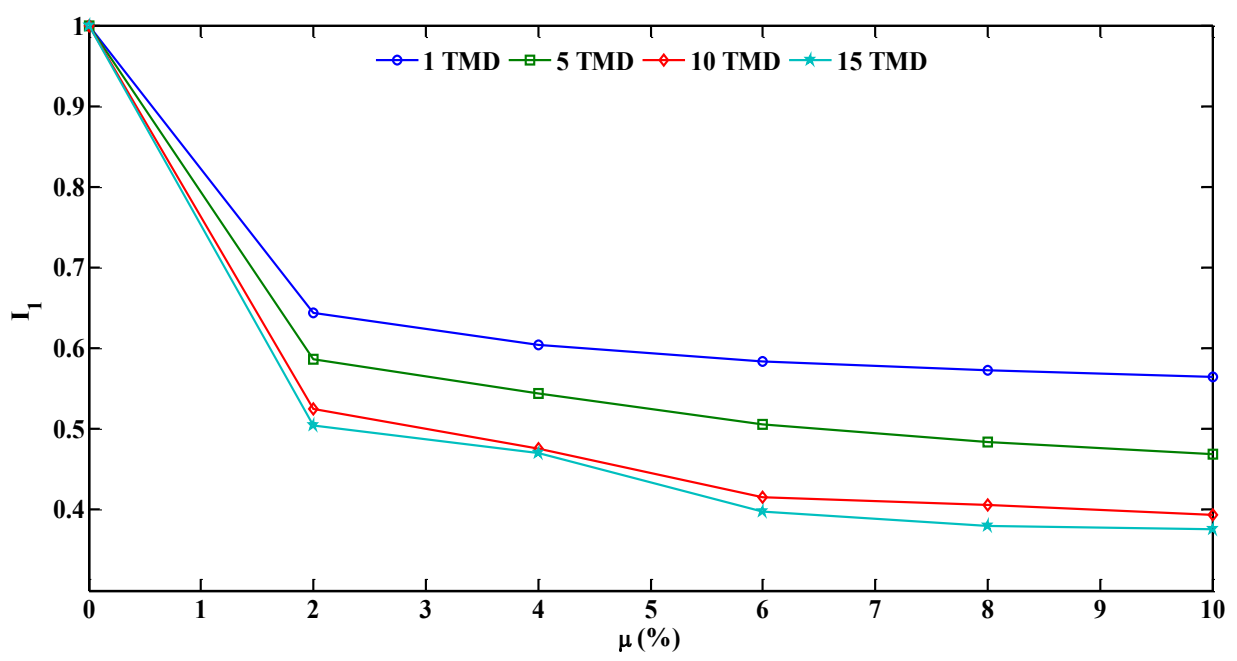

Fig. 6: Effect of number of and mass ratio of TMDs on the value of I1 index

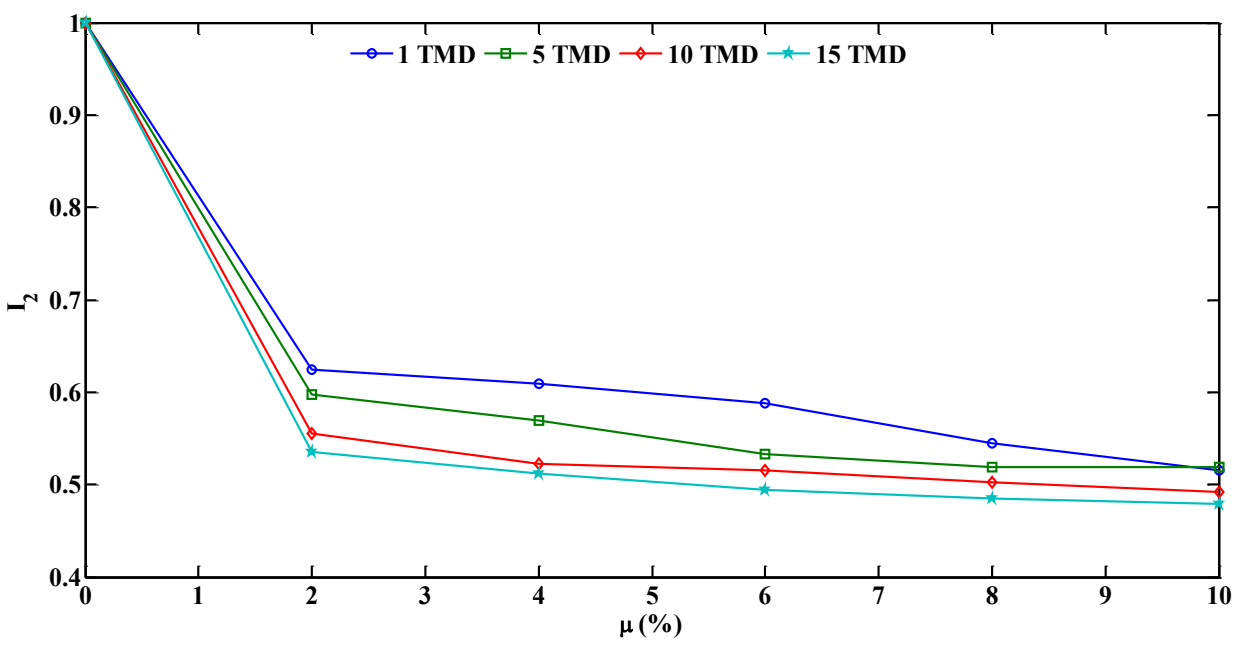

Fig. 7: Effect of number of and mass ratio of TMDs on the value of I 2 index 


\section{Conclusion}

In this study, an effective way has been proposed to design MTMD to reduce the seismic response of structures that is based on defining a multi-objective optimization problem and to solve it cuckoo multi-objective optimization algorithms is used. Since MTMD is a multi-objective optimization problem, three objective criteria including minimizing the maximum displacement of roof, maximum acceleration of roof and TMD maximum displacement of roof are considered in the optimization problem. The results of numerical simulations for a shear frame of ten floors exposed to earthquakes show that CMOA is able to offer appropriate solutions in the form of Parato charts for setting optimal parameters of MTMD, based on the objectives of the designer, which is because of creating a proper compromise among the objective functions in conflict with each other.

In addition, the results show that MTMD desirably reduce seismic response of structures; the results also show that MTMD performance depends on input quake, TMD mass ratio and the number of TMDs. MTMD performance to input earthquake depends on mass ratio of TMD and the number of TMDs. TMD mass ratio increase to a certain extent reduces the seismic response and then has no big impact on responses. This result has also been obtained for the number of TMDs. In the studied structures by increase in TMDs mass ratio to $6 \%$, displacement and acceleration of roof reduces and for more than $6 \%$, decline process has not greatly altered. It is also seen that for a fixed mass ratio increasing TMDs from 1 to 10 leads to a good reduction in $I_{1}$ and $I_{2}$ values, but with more increase of TMDs the response has not changed much.

\section{Data Availability Statement}

Some or all data, models, or code generated or used during the study are available from the corresponding author by request.

\section{Acknowledgement}

We thank Water Engineering Department, College of Agriculture, Isfahan university of Technology and Isfahan, Iran. Postal Code: 84156-83111.

\section{Funding Information}

Funding information is not applicable. No funding was received. No grants were received.

\section{Author's Contributions}

All authors designed the study, collected data, wrote the manuscript and revised it.

\section{Ethics}

The present Study and ethical aspect was approved by Water Engineering Department, College of Agriculture, Isfahan University of Technology and Isfahan, Iran. Postal Code: 84156-83111.

\section{References}

Abé, M. and Fujino, Y. (1994). Dynamic characterization of multiple tuned mass dampers and some design formulas. Earthquake engineering and structural dynamics, 23(8), 813-835.

Civicioglu, P. and Besdok, E. (2013). A conceptual comparison of the Cuckoo-search, particle swarm optimization, differential evolution and artificial bee colony algorithms. Artificial intelligence review, 39(4), 315-346.

Dehghan-Niri, E., Zahrai, S. M. and Mohtat, A. (2010). Effectiveness-robustness objectives in MTMD system design: An evolutionary optimal design methodology. Structural Control and Health Monitoring: The Official Journal of the International Association for Structural Control and Monitoring and of the European Association for the Control of Structures, 17(2), 218-236.

Fan, F. G. and Ahmadi, G. (1990). Nonstationary kanaitajimi models for el centro 1940 and mexico city 1985 earthquakes. Probabilistic Engineering Mechanics, 5(4), 171-181.

Gandomi, A. H., Talatahari, S., Yang, X. S. and Deb, S. (2013). Design optimization of truss structures using cuckoo search algorithm. The Structural Design of Tall and Special Buildings, 22(17), 1330-1349.

Hoang, N. and Warnitchai, P. (2005). Design of multiple tuned mass dampers by using a numerical optimizer. Earthquake engineering and structural dynamics, 34(2), 125-144.

Igusa, T. and Xu, K. (1994). Vibration control using multiple tuned mass dampers. Journal of sound and vibration, 175(4), 491-503.

Jangid, R. S. (1999). Optimum multiple tuned mass dampers for base-excited undamped system. Earthquake engineering and structural dynamics, 28(9), 1041-1049.

Kareem, A. and Kline, S. (1995). Performance of multiple mass dampers under random loading. Journal of structural engineering, 121(2), 348-361.

Li, C. (2002). Optimum multiple tuned mass dampers for structures under the ground acceleration based on DDMF and ADMF. Earthquake engineering and structural dynamics, 31(4), 897-919.

Moon, K. S. (2010). Vertically distributed multiple tuned mass dampers in tall buildings: performance analysis and preliminary design. The Structural Design of Tall and Special Buildings, 19(3), 347-366. 
Nagarajaiah, S. and Narasimhan, S. (2006). Smart base-isolated benchmark building. Part II: phase I sample controllers for linear isolation systems. Structural Control and Health Monitoring: The Official Journal of the International Association for Structural Control and Monitoring and of the European Association for the Control of Structures, 13(2-3), 589-604.

Ohtori, Y., Christenson, R. E., Spencer Jr, B. F. and Dyke, S. J. (2004). Benchmark control problems for seismically excited nonlinear buildings. Journal of engineering mechanics, 130(4), 366-385.

Rajabioun, R. (2011). Cuckoo optimization algorithm. Applied soft computing, 11(8), 5508-5518.

Tigli, O. F. (2012). Optimum vibration absorber (tuned mass damper) design for linear damped systems subjected to random loads. Journal of sound and vibration, 331(13), 3035-3049.
Wu, J. and Chen, G. (2000, June). Optimization of multiple tuned mass dampers for seismic response reduction. In Proceedings of the 2000 American Control Conference. ACC (IEEE Cat. No. 00CH36334) (Vol. 1, No. 6, pp. 519-523). IEEE.

Yamaguchi, H. and Harnpornchai, N. (1993). Fundamental characteristics of multiple tuned mass dampers for suppressing harmonically forced oscillations. Earthquake engineering and structural dynamics, 22(1), 51-62.

Yang, X. S. and Deb, S. (2009, December). Cuckoo search via Lévy flights. In 2009 World congress on nature and biologically inspired computing (NaBIC) (pp. 210-214). IEEE.

Yang, X. S. and Deb, S. (2014). Cuckoo search: recent advances and applications. Neural Computing and Applications, 24(1), 169-174. 